\title{
Integrating PrEP delivery in public health family planning clinics: a protocol for a pragmatic stepped wedge cluster randomized trial in Kenya
}

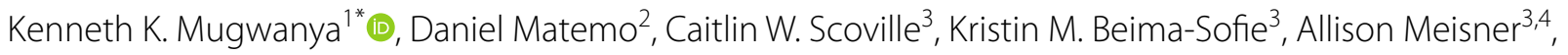
Dickens Onyango ${ }^{5}$, Mary Mugambi ${ }^{6}$, Erika Feutz ${ }^{3}$, Cole Grabow ${ }^{3}$, Ruanne Barnabas ${ }^{7}$, Bryan Weiner ${ }^{3}$, Jared M. Baeten ${ }^{8,9}$, John Kinuthia ${ }^{10}$ and for the FP Plus Team

\begin{abstract}
Background: Adolescent girls and young women account for a disproportionate fraction of new HIV infections in Africa and are a priority population for HIV prevention, including provision of pre-exposure prophylaxis (PrEP). Anchoring PrEP delivery to care settings like family planning (FP) services that women already access routinely may offer an efficient platform to reach HIV at-risk women. However, context-specific implementation science evaluation is needed.

Methods: The Family Planning Plus Project is a prospective, pragmatic implementation evaluation, designed as a stepped wedge, cluster randomized trial, at 12 clinics in Kenya. In collaboration with the Kenya Ministry of Health and Kisumu County Department of Health, we will introduce integration of HIV risk screening and PrEP delivery in public health FP clinics. The core multifaceted implementation strategies to integrate PrEP in FP clinics will include: (1) PrEP delivery by existing FP clinic staff, (2) health provider training, (3) PrEP technical assistance to coach and mentor providers, (4) joint supervision with Kisumu County health officials, and (5) stakeholder engagement. All core components of PrEP delivery -including screening for HIV risk, HIV testing, dispensing, adherence and risk reduction counseling, assessment of side effects, and provision of refills, or safety assessment-will be conducted by existing FP clinic staff as part of a standard care service package. The goal is to catalyze sustainable scale-up within existing infrastructures beyond the project. We will rigorously evaluate implementation outcomes and impact, using the RE-AIM (Reach, Effectiveness, Adoption, Implementation, Maintenance) framework, and we will use Organizational Readiness for Implementing Change (ORIC) and the Consolidated Framework for Implementation Science Research (CFIR) to assess readiness to implement and contextual enablers and barriers of implementation, including how clinics innovate efficient delivery systems.
\end{abstract}

Discussion: Anchoring PrEP delivery to existing FP systems and staffing has tremendous potential to address barriers that women face in accessing HIV prevention and PrEP care, including lack of time, cost, and stigma of visiting a facility solely for HIV prevention. The FP Plus Project will initiate preparation for full-scale and sustainable model of

*Correspondence: mugwanya@uw.edu

${ }^{1}$ Departments of Global Health and Epidemiology, University of Washington, 325 Ninth Avenue, \#HMC 359927, Seattle, WA 98104, USA

Full list of author information is available at the end of the article permits use, sharing, adaptation, distribution and reproduction in any medium or format, as long as you give appropriate credit to the original author(s) and the source, provide a link to the Creative Commons licence, and indicate if changes were made. The images or other third party material in this article are included in the article's Creative Commons licence, unless indicated otherwise in a credit line to the material. If material is not included in the article's Creative Commons licence and your intended use is not permitted by statutory regulation or exceeds the permitted use, you will need to obtain permission directly from the copyright holder. To view a copy of this licence, visit http://creativecommons.org/licenses/by/4.0/. The Creative Commons Public Domain Dedication waiver (http://creativeco mmons.org/publicdomain/zero/1.0/) applies to the data made available in this article, unless otherwise stated in a credit line to the data. 
integration of comprehensive HIV prevention services, including PrEP implementation, in public health FP clinics in low-income settings.

Trial registration

Registered with ClinicalTrials.gov on December 14, 2020: NCT04666792

\section{Contributions to the literature}

- Young African women are a priority for HIV prevention including PrEP provision because they account for a disproportionate percentage of new HIV infections.

- Routine family planning clinics are attractive settings for PrEP implementation due to broad coverage for sexually active women. However, there is limited realworld evidence on effective strategies for integrated family planning and PrEP delivery in African settings.

- Rigorous implementation science evaluations from this work will provide important information on how to effectively and sustainably reach at-risk women for PrEP in resource-limited settings. The findings will also be valuable in translating emerging HIV prevention modalities into real-world implementation.

\section{Background}

Women account for a disproportionate percentage of individuals with new HIV infections in Africa and are a priority population for HIV prevention [1], including provision of pre-exposure prophylaxis (PrEP). PrEP is a highly effective user-controlled prevention method, with potential to reduce incident HIV infections in Africa if delivered with sufficient coverage among at-risk populations [2-4]. In many settings in Africa, family planning (FP) clinics provide broad coverage for women in their reproductive years [5] and offer an opportunity to integrate HIV prevention and sexual and reproductive health services (SRH), including PrEP provision and management of sexually transmitted infections (STIs). A recent large clinical trial of contraceptive use and HIV acquisition (ECHO Trial) found that HIV risk was alarmingly high for women seeking family planning [6]. These results have emphasized the need to strengthen integration of HIV prevention and FP services. The World Health Organization (WHO) has issued a renewed call-to-action for efforts and commitment to support countries with high HIV incidence rates, to develop plans to provide integrated FP and HIV and STI services [7].

In the recently completed PrEP Implementation in Young Women and Adolescent (PrIYA) program (funded through PEPFAR DREAMS innovation challenge), we pioneered PrEP provision in FP clinics and demonstrated feasibility of integrating PrEP provision in FP systems using project-dedicated staff embedded in FP clinics [8]. Over 1271 women seeking FP services were screened for HIV risk factors across eight FP clinics in Kisumu County, Kenya with 22\% PrEP uptake in during a 12-month program period. However, PrEP uptake declined when project-dedicated staff left after the end of the program, demonstrating the need for sustainable PrEP delivery models that fully integrate into existing staff and clinic systems. With more efficient PrEP integration, there is opportunity to optimize women-centered care with a one-stop approach to facility-based FP and HIV prevention care. Here, we describe the design and the application of the Reach, Effectiveness, Adoption, Implementation, and Maintenance (RE-AIM), Organizational Readiness for Implementing Change (ORIC), and the Consolidated Framework for Implementation Science Research (CFIR) frameworks to a programmatic steppedwedge evaluation to integrate PrEP in Kenyan public health FP systems (the FP Plus Project). Triangulation of data from multiple sources guided by these frameworks will help to characterize key processes needed for the development of sustainable integration of FP and HIV prevention services.

\section{Methods}

\section{Overall goal and specific aims}

The overall goal of the FP Plus Project is to catalyze integration of comprehensive screening for HIV risk and PrEP delivery in public health FP clinics in Kenya. The specific aims are:

1) Facilitate integration of comprehensive screening for HIV risk behaviors and delivery of PrEP in public FP clinics and evaluate program impact.

2) Assess clinic readiness to implement PrEP, fidelity to PrEP service delivery, impact on current services, and facilitators and barriers to integrating PrEP delivery in FP clinics

3) Assess incremental costs, budget impact, and affordability of integrating PrEP delivery in FP clinics

4) Refine and optimize simple data systems that will expand and support delivery of integrated FP and HIV prevention services at scale. 


\section{Population, Setting, and Facility selection}

The population includes sexually active HIV-uninfected women of reproductive years accessing FP services in the Kisumu area of Kenya, given it is a high HIV burden area [9]. The project is conducted at 12 facilities selected through a joint decision process by project investigators and Kisumu County officials. Clinic selection was based on patient volume, readiness to participate, and catchment area to capture the diversity that represent the geographic, economic, and demographic characteristics of all women accessing FP services and breadth among the clinic staff.

Eligibility to participate in the program will reflect the real-world implementation nature of this work. Thus, the program will be set up for all women of reproductive age ( $\geq 15$ years), HIV uninfected or of unknown HIV status receiving routine FP services. The broad eligibility criteria is designed to align with the public health nature of this work; to operate effectively, efficiently, and ethically in community settings, PrEP will need to be broadly accessible to all at-risk adolescent girls and young women who shoulder the biggest burden of HIV infections in this setting. Determination of eligibility for PrEP will be conducted according to the
Kenya national PrEP guidelines. All women determined to be at risk for HIV, or expressing interest in PrEP, will have access to PrEP medication free of charge as part of the Kenya national PrEP scale-up program.

\section{Project Design and Intervention}

The FP Plus Project is a prospective, pragmatic stepped wedge cluster randomized trial (SWCRT). In collaboration with the Kenya MOH and Kisumu County department of health, systematic screening for HIV risk and counseling for and provision of PrEP will be introduced and actively promoted in FP clinics in three staged successive waves (steps), occurring approximately every three months with an average of $60 \mathrm{HIV}$-uninfected women accessing services per clinic per step, and four clinics initiating implementation per step (Fig. 1). We will use steps four to ten (Fig. 1) to refine and optimize the intervention with varying ongoing technical assistance and supervision intensity to understand and document implementation process and outcomes and how effectively delivery of integrated PrEP services is institutionalized in FP clinics.

\begin{tabular}{|c|c|c|c|c|c|c|c|c|c|c|c|}
\hline Group & Baseline & Step 1 & Step 2 & Step 3 & Step 4 & Step 5 & Step 6 & Step 7 & Step 8 & Step 9 & Step 10 \\
\hline Clinic 1 & $\mathrm{~s}$ & 1 & 1 & 1 & I & M & M & M & M & M & M \\
\hline Clinic 2 & $\mathrm{~s}$ & 1 & 1 & 1 & 1 & M & M & M & M & M & M \\
\hline Clinic 3 & $\mathrm{~s}$ & 1 & 1 & 1 & 1 & M & M & M & M & M & M \\
\hline Clinic 4 & $s$ & 1 & I & I & 1 & M & M & M & M & M & M \\
\hline Clinic 5 & S & $S$ & 1 & 1 & 1 & 1 & $M$ & $M$ & $M$ & $M$ & $M$ \\
\hline Clinic 6 & s & $s$ & 1 & 1 & I & 1 & M & $M$ & $M$ & $M$ & $M$ \\
\hline Clinic 7 & $\mathrm{~s}$ & $s$ & 1 & 1 & 1 & 1 & $M$ & M & $M$ & M & M \\
\hline Clinic 8 & 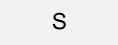 & $s$ & 1 & I & I & 1 & $M$ & $M$ & $M$ & $M$ & M \\
\hline Clinic 9 & S & S & S & 1 & 1 & 1 & 1 & $M$ & M & $M$ & $M$ \\
\hline Clinic 10 & $\mathrm{~s}$ & $s$ & s & 1 & I & 1 & 1 & M & M & M & M \\
\hline Clinic 11 & $s$ & $s$ & $s$ & $\mathrm{I}$ & 1 & 1 & 1 & M & M & M & M \\
\hline Clinic 12 & s & $s$ & $s$ & 1 & I & I & 1 & M & $\mathrm{M}$ & M & $M$ \\
\hline \multicolumn{12}{|c|}{$\begin{array}{l}\text { Schema of stepped wedge study design. " } \mathbf{S} \text { " indicates current/standard of care, "I" indicates intervention implementation (Intensive (i.e., training, TA, Quarterly } \\
\text { Joint supervision with Kisumu County), and "M" indicates maintenance phase, after the intensive intervention activities have been scaled back. Each step is } \\
\text { 12 weeks long, with } 2 \text { weeks of training before each step where the intervention is introduced (Steps } 1-3 \text { ). Each clinic will have at least } 12 \text { months of intensive } \\
\text { intervention implementation (I) and at least } 12 \text { months of maintenance phase }(\mathbf{M}) \text {. Statistical evaluation of the primary intervention effectiveness will be based } \\
\text { on the baseline and Steps } 1-3 \text {. Implementation outcomes including during maintenance phase will be evaluated using I and } \mathbf{M} \text { phases. }\end{array}$} \\
\hline
\end{tabular}

Fig. 1 Schematic of stepped-wedge cluster randomized trial design. 


\section{Randomization}

Randomization was carried out at the beginning i.e., a single point in time (at a public virtual meeting with the Kisumu County Health leadership and health facility representatives), randomizing the order with which the clinics (clusters) cross over from usual care to implementation of the intervention package. Randomization was conducted without restriction and implemented in two-stages using an online wheel-of- fortune. First, clinics were assigned to three groups of four clinics each and second, the groups were then assigned to the order (i.e., order of steps) to which they would start implementation (Fig. 1).

\section{Intervention: Implementation strategies to integrate PrEP services in FP clinics}

For this implementation project, the intervention to integrate PrEP in Kenyan FP clinics is a multifaceted implementation strategy with the following core components: 1) promotion of comprehensive screening for HIV risk and PrEP eligibility by existing FP staff (including HIV testing, HIV risk screening, male partner testing, PrEP provision, and adherence and safety counseling); 2) Training of existing health care providers; 3) PrEP technical assistance to coach and mentor health providers; and 4) Joint supervision with Kisumu County health officials. At all implementing clinics, we will conduct a preimplementation needs assessment to understand and document current processes on HIV risk screening and testing, existing PrEP screening and provision (expected to be minimal), STI screening, testing and treatment, and male partner testing. Prior to initiating implementation at each clinic, we will conduct clinic-wide training for health care providers, and we will engage existing FP providers in ongoing continuous quality improvement to optimize PrEP delivery. Clinics will start implementation after two weeks of intensive training. Each clinic will have at least 12 months of intensive implementation of the intervention package followed by at least 12 months of maintenance phase (Fig. 1)

\section{Screening for HIV risk and PrEP provision}

We will optimize integration of comprehensive HIV prevention services and PrEP delivery using existing FP clinic providers within existing infrastructure. All core components of PrEP delivery-including screening for HIV behavior risk, HIV testing, dispensing, adherence and risk reduction counseling, assessment of side effects, provision of PrEP refills, and safety assessments - will be conducted by existing FP clinic staff as part of the standard of care service package. The goal is to catalyze scale-up within existing clinic infrastructure that is sustainable beyond the study. As part of standard of care services, clinics will promote comprehensive provision of integrated FP services and HIV prevention services, including contraception methods use and preferences, promotion of knowledge of partner HIV status, condom provision, and screening and treatment of STIs. Determination of eligibility for PrEP, clinical provision of PrEP, and follow-up of clients who initiate PrEP will be conducted according to the Kenya national PrEP guidelines using $\mathrm{MOH} / \mathrm{NASCOP}$ tools, which include a risk assessment screening tool and clinical encounter form [10]. The Kenya PrEP guidelines identify the presence of any of the following behavioral factors in the last six months as indication for substantial ongoing risk of acquiring HIV: 1) Inconsistent or no condom use, 2) Having high risk sex partner(s) and of unknown HIV status, 3) Engaging in transactional sex, 4) History of ongoing intimate partner and gender-based violence, 5) Recent sexually transmitted infections- self-reported or etiologically diagnosed, 6) Recurrent use of post-exposure prophylaxis, 7) Recurrent sex under the influence of alcohol/recreational drugs; 8) Injection drug use with shared needles and/or syringes, and 9) Having an HIV positive partner. Per the Kenya MOH PrEP guidelines, persons who initiate PrEP have follow up visits at one month and then quarterly after initiation, with HIV testing, ongoing behavior risk assessment, and adherence and safety counseling.

PrEP medications and commodities will be provided by the Kenya national stock of medications from the Kenya Medical Supply Authority as part of the national PrEP scale-up program. PrEP has regulatory and normative guidance sanctions in Kenya and is defined as part of standard care, in part based on a series of clinical trials and implementation studies done in Kenyan populations [11-20]. All women determined to be at risk for HIV, or expressing interest in PrEP, will receive PrEP medication from FP clinics free of charge as part of the Kenya national PrEP scale-up program.

\section{Provider training to strengthen capacity for PrEP implementation}

The program will work with the $\mathrm{MOH} / \mathrm{NASCOP}$ and Kisumu County Health Team to support clinics' readiness to deliver PrEP in a combination HIV prevention package in FP clinics. Provider training will be conducted at each of the participating clinics (in-facility training) using the approved case-based interactive Kenya MOH/ NASCOP PrEP training curriculum [21]. Content specific to FP clinics will be added, including topics on FP, STI, treatment, and partner testing services with the goal of equipping providers with the comprehensive knowledge and skills to provide integrated FP and combination HIV prevention services, including PrEP services. The training will be conducted at each facility and curriculum 
modules will be delivered over a 2-week period immediately prior to implementation. A Continuous Medical Education-like refresher training will be delivered quarterly following implementation. Clinics will begin PrEP delivery within two weeks of training.

\section{PrEP technical assistance}

We will conduct ongoing technical assistance to clinics using trained experienced program nurses to coach, mentor, and guide FP clinics to troubleshoot emerging challenges with integrating new services. Technical advisors (TAs) will be project nurses with training in PrEP delivery. TAs will conduct structured periodic visits to each clinic to engage with the health care providers and observe and document PrEP implementation processes, with a specific focus on delivery modifications over time to understand integration optimization within and between facilities. Using rapid-cycle analysis approaches, TAs will make summary reports at the end of each clinic visit highlighting what changed, why it changed, who initiated the changes, and outcome of any changes instituted. Best practices will be shared across all clinics (cross-clinic pollination) for possible adoption. The project will work with the County Health team and implementing clinics to optimize simple data systems that include indicators for screening HIV risk and PrEP provision.

\section{Joint supervision with Kisumu County health team}

At each clinic, the TAs will visit the clinic weekly for first three months, and then complete monthly visits through the 12-month intensive implementation phase. During the maintenance phase, TAs will complete quarterly visits or as requested by the implementing clinics focused on understating institutionalization of integration. Every three months throughout the first 18 months of intervention implementation (i.e., 12 months of intense implementation and at least 6 months of maintenance), the TAs will be accompanied to the facility visits by County health and or Kenya MOH/NASCOP supervisors to audit for process and integration indictors for FP services, HIV prevention services, and PrEP and progress.

\section{Sample size and power}

The co-primary outcomes will be the proportion of women screened for HIV risk and PrEP eligibility and the number and proportion of women eligible women who initiate PrEP. The study has been powered for the PrEP eligibility screening outcome. The denominator will consist of all HIV uninfected women accessing services at the implementing FP clinic during each step (time period). Although screening for HIV risk behaviors is an expected service in FP clinics, it is not routinely done or documented in most FP settings. After the intervention, we expect to see screening for HIV risk and PrEP among general population women to increase from $\sim 5 \%$ to $25 \%$ (mean $\sim 20 \%$ ). We used an intracluster correlation coefficient value of 0.15 , which is generally considered conservative in this context, and we conservatively allowed the proportion of HIV-uninfected women counseled on HIV risk and PrEP to be $6 \%$ in the absence of intervention at the end of the study (i.e., a $20 \%$ increase in proportion counseled due to factors outside of the intervention). Given these parameters, if we enroll 56 HIV-uninfected women in each of the 12 clinics during each of the first four steps (including the baseline step), where four clinics initiate the intervention in each of first three post-baseline steps (yielding 2688 total participants across the 12 clinics and the first four steps), we would have $80 \%$ power. Note that these calculations provide the number of HIV-uninfected women required; the number of women needing to be screened would be higher (and would depend on the HIV positivity rate). To fully understand how clinics build new efficient systems for delivery of integrated FP and HIV prevention services, we will study data on a total of at least 5000 women accessing routine services across the $12 \mathrm{FP}$ clinics. This sample size will permit a full-scale test of the system, since implementation outcomes and the efficiency in delivery are to a greater part at the system level, above and beyond the individual client encounter.

\section{Data collection, measurement, and evaluation of process and implementation outcomes}

We will use multiple data sources (Table 1) to robustly synthesize and triangulate the process of integrating PrEP delivery in public health FP clinics and to identify contextually relevant strategies for successful implementation. Data sources will include: 1) Abstraction of program data, 2) Qualitative interviews (policy key informants, healthcare providers, women), 3) Quantitative questionnaires (healthcare providers, women), 4) TA visit summaries and rapid cycle debrief reports, 5) Patient actors, 6) Clinic and client flow mapping, 7) Activity-based costing and time and motion studies; 8) individual-level clinical data from the nested observational cohort, and 9) Random blood draw for adherence (Table 1).

\section{Abstraction of program data}

Clinical records in FP clincs are documented on standardized MOH FP register which collects program data on age, contraception method use, women HIV status, and cervical counseling. In collaboration with the Kisumu county health team, new indicators on screening for HIV risk and PrEP eligibility as part of our goal to streamiline simple but scalable HIV porevention program indicators. 
Table 1 Data sources

\begin{tabular}{|c|c|c|}
\hline Data source & Description & Purpose \\
\hline Data abstraction & - Data abstracted from clinical delivery tools & $\begin{array}{l}\text { - Define profile of screened and initiated on PrEP } \\
\text { and whether persons are appropriately put on } \\
\text { PrEP }\end{array}$ \\
\hline $\begin{array}{l}\text { Technical assistance reports and rapid cycle } \\
\text { debriefs. }\end{array}$ & $\begin{array}{l}\text { - Rapid cycle debriefs and TA reports prepared } \\
\text { at baseline and 6-monthly }\end{array}$ & $\begin{array}{l}\text { - Document detailed knowledge of process of } \\
\text { integration of PrEP delivery and track changes in } \\
\text { PrEP implementation processes } \\
\text { - Rapid cycle analysis to convey to facilities for } \\
\text { quality improvement }\end{array}$ \\
\hline $\begin{array}{l}\text { Qualitative interviews: women, healthcare } \\
\text { providers, and policy key informants }\end{array}$ & $\begin{array}{l}\text { - Purposefully sampled client and key inform- } \\
\text { ants involved in the delivery }\end{array}$ & $\begin{array}{l}\text { - Characterize process of adoption and integra- } \\
\text { tion of PrEP delivery and track changes in PrEP } \\
\text { implementation processes, including barriers and } \\
\text { facilities }\end{array}$ \\
\hline Quantitative surveys & $\begin{array}{l}\text { - Healthcare providers } \\
\text { - Random cross-sectional exit surveys with } \\
\text { women at the end of clinic visit }\end{array}$ & $\begin{array}{l}\text { - Assess acceptability, clinics' readiness to imple- } \\
\text { ment, clinic inner settings } \\
\text { - HIV and STI risk perception, characteristics of } \\
\text { women accessing through FP clinics, stigma, and } \\
\text { satisfaction with services offered. }\end{array}$ \\
\hline $\begin{array}{l}\text { Activity-based costing, and time and motions } \\
\text { studies }\end{array}$ & - Primary data collection & $\begin{array}{l}\text { - Activity-based costing, and time and motions } \\
\text { studies }\end{array}$ \\
\hline Clinic and client flow mapping & - Primary data collection & $\begin{array}{l}\text { Establish baseline flow, track how new services } \\
\text { are incorporated in the flow and bottlenecks, and } \\
\text { document patient wait-time }\end{array}$ \\
\hline Standardized patient actors & $\begin{array}{l}\text { - Standardized clients make unannounced visit } \\
\text { to subset of clinics and complete a checklist }\end{array}$ & $\begin{array}{l}\text { - Assess implementation fidelity by document- } \\
\text { ing type of services offered, quality of services } \\
\text { received, and provider attitudes }\end{array}$ \\
\hline Random blood draw & $\begin{array}{l}\text { Dried blood spots collected at } \sim 10 \% \text { visits on } \\
\text { persons using PrEP }\end{array}$ & $\begin{array}{l}\text { Objective assessment of PrEP adherence (teno- } \\
\text { fovir levels) }\end{array}$ \\
\hline Nested observational cohort & $\begin{array}{l}\text { - Women identified as eligible for PrEP, both } \\
\text { who initiate and those who decline }\end{array}$ & $\begin{array}{l}\text { Detailed individual-level outcomes: HIV risk } \\
\text { behaviors, HIV risk perception, HIV incidence, HIV } \\
\text { prevention decision making, contraception use, } \\
\text { stigma, STI burden, PrEP retention, and reasons for } \\
\text { discontinuation }\end{array}$ \\
\hline
\end{tabular}

For persons who initiate PrEP, Kenya $\mathrm{MOH}$ clinical encounter form (PrEP Card) is used for clinical monitoring and program evaluation. Dedicated project staff will weekly abstract program data (e.g., number women screened for HIV risk and PrEP initiations) and individual clinical records from these standard $\mathrm{MOH}$ tools. Individual data will be used to characterize PrEP screening, PrEP eligibility and initiation, HIV risk profiles of PrEP initiators, self-reported adherence, retention, HIV infection, and side effects.

\section{Application of the RE-AIM framework}

We will conduct rigorous mixed-methods evaluation using the RE-AIM framework to gain a comprehensive understanding of how effectively PrEP implementation is integrated in FP systems and program impact (Table 2). The RE-AIM framework guides evaluation of the public health impact of complex interventions and can provide actionable information on which to base ongoing and post-trial expansion planning. For this project, Reach will be defined as the proportion and subgroups of women accessing FP services who are screened for and uptake
PrEP; Effectiveness will be assessed at individual level as 1) PrEP adherence, quantified by tenofovir levels in dried blood spots, and 2) women staying HIV free; Adoption will be assessed at the clinic and provider level defined as the proportion of 1) clinics approached who implement the intervention 2) targeted providers who are trained and implement PrEP thereafter; Fidelity to PrEP delivery will be assessed as proportion of core components delivered per Kenya PrEP guidelines; and Maintenance, will be the number of clinics delivering PrEP at 6 and 12 months after the intensive technical assistance

\section{Clinic readiness to implement PrEP in FP clinics}

At each clinic prior to implementation, we will administer a validated organizational readiness for change ORIC psychometric tool [22] to clinic managers and providers to assess for determinants of readiness to implement, including provider knowledge and confidence to provide PrEP, perception of resource availability, and situational factors including timing of the program. We will define facets of readiness (i.e., change commitment and change efficacy), their immediate determinants, and how they 
Table 2 Application of the RE-AIM framework to evaluate integration of PrEP delivery in into public health FP clinics

\begin{tabular}{|c|c|c|}
\hline Domain & Measurement level & Project specific outcome measures \\
\hline \multirow[t]{2}{*}{ Reach } & Individual & $\begin{array}{l}\text { - Proportion of women screened for HIV risk and PrEP } \\
\text { - Number and proportion of at-risk persons initiated on PrEP }\end{array}$ \\
\hline & Clinic & - Characteristics of implementing clinics \\
\hline \multirow[t]{2}{*}{ Effectiveness } & Individual & $\begin{array}{l}\text { - Incident HIV infection among PrEP users } \\
\text { - Detectable tenofovir levels in randomly collected DBS from PrEP users } \\
\text { - Client opportunity costs (e.g., clinic wait time) }\end{array}$ \\
\hline & Program & - Cost and budget impact from the pragmatic perspective \\
\hline \multirow[t]{2}{*}{ Adoption } & Individual & - PrEP continuation \\
\hline & Clinic & $\begin{array}{l}\text { - Number and } \% \text { of clinics initially approached implementing PrEP in FP clinics } \\
\text { - Number and } \% \text { of trained clinical FP staff who delivered PrEP at least once }\end{array}$ \\
\hline \multirow[t]{2}{*}{ Implementation } & Provider & $\begin{array}{l}\text { - Number and \% of trained appropriately initiated on PrEP } \\
\text { - Consistency of implementation across staff (assessed by patient actors) } \\
\text { - Opportunity costs (e.g., Workload, impact on other services) }\end{array}$ \\
\hline & Clinic & $\begin{array}{l}\text { - Number of clinics regularly completing MOH PrEP M \& E tools and report } \\
\text { PrEP } \\
\text { - Number and proportion of clinics in which screening for HIV risk and PrEP } \\
\text { regularly documented. } \\
\text { - Proportion of women testing is documented }\end{array}$ \\
\hline \multirow[t]{2}{*}{ Maintenance } & Individual & - 3- and 6-month PrEP continuation rates \\
\hline & Clinic & $\begin{array}{l}\text { - Number of FP clinics implementing PrEP FP } 6 \text { months, } 12 \text { months after scale } \\
\text { back of technical assistance } \\
\text { - Number of clinics with PrEP listed in the service charter }\end{array}$ \\
\hline
\end{tabular}

impact implementation of PrEP. We will use this data to understand clinic-level variations in implementation performance at both high and low performing clinics, and how they affect implementation outcomes.

\section{Clinic and client flow mapping}

We will conduct workflow mapping at all sites to identify potential bottlenecks, differences and commonalities among sites at baseline and annually. We will solicit provider engagement in defining new flow maps to accommodate new tasks and patient flows. Current and future flow maps will be created for each site to demonstrate new pathways.

\section{Qualitative interviews to understand delivery}

We will prospectively conduct qualitative interviews to rigorously understand key influences on integration of HIV prevention and PrEP in FP clinics, at the level of individual women (clients), healthcare providers, the organization (i.e., clinic), and the health system (policy key-informants). Interviews will use the Consolidated Framework for Implementation Science Research (CFIR) [23] to guide development of data collection instruments, data analysis, and reporting of findings.

Qualitative interviews with women will assess women's experiences accessing and using (or not using) HIV prevention services, including PrEP in FP clinics, confidence in the health system, decision-making processes, partnership dynamics, and stigma and community norms around
HIV and FP. Because our overarching goal is development of a sustainable HIV prevention program broadly, not specific to PrEP, stratified purposive sampling will be used to ensure that the overall sample represents relevant sub-populations of women including [24, 25]: 1) women who have risk factors for HIV but choose not to initiate PrEP, 2) women who choose to initiate PrEP, and 3) women who initiate PrEP but later discontinue use.

Qualitative interviews with healthcare providers at FP clinics (clinic managers, counselors, nurses, doctors) and policy key informants (national level and County level) will be conducted during baseline, early implementation and maintenance phases to understand contextual enablers and barriers of implementation acceptability, feasibility, and fidelity. We will specifically evaluate knowledge and confidence delivering PrEP, perception of the impact of PrEP delivery on current FP service provision, and suggestions for efficient integration approaches in the context of FP clinics. Analysis of personal experiences with integrated FP and PrEP delivery will be used to identify determinants of successful implementation and innovative strategies that can be harnessed for future scale-up.

\section{Quantitative surveys}

We will use quantitative tools including cross-sectional exit surveys among women to complement qualitative interviews. Surveys will focus on understanding HIV risk perceptions, services offered or expected to be offered, quality of care and women satisfaction with services 
received, and women's experiences with the health systems. Cross-sectional surveys will be conducted during baseline period and at least annually by project dedicated staff, as clients exit the facility. These surveys will help obtain important data that cannot be obtained from program tools. We will also use a quantitative tool for CFIR inner settings [26] with clinic-managers, in-charges, and frontline healthcare providers during intervention implementation phase to gain deep understanding of cliniclevel factors that impact implementation. Data from provider surveys will provide further insights into characteristics of low and high performing clinics.

Standardized patient actors to assess implementation fidelity We will prospectively document the extent to which the core components of the PrEP implementation are implemented as planned. We will examine fidelity across dimensions of adherence, exposure, quality of delivery, provider responsiveness, and program differentiation. We will assess and document factors that affect fidelity, including characteristics of providers, clinics, women, the FP or community context, and program support systems (e.g., County supervision, technical assistance). Specifically, we will use unannounced standardized patient actors to measure fidelity of the implementation of comprehensive HIV prevention services, including PrEP delivery (i.e., counseling and screening for HIV risk factors, HIV testing, PrEP provision). Patient actors will visit a subset of clinics for two consecutive visits, once to screen for HIV risk and PrEP, and a second time to refill PrEP. At the end of each unannounced visit, the standardized patient will complete a checklist to document offered services, quality of services received, provider attitudes, and visit duration.

\section{Random blood draw to evaluate PrEP adherence}

Among women who initiate PrEP, adherence will be objectively evaluated by tenofovir levels measured from dried blood spots collected at a subset of randomly selected visits ( $10-20 \%)$. Adherence will also be measured using pharmacy refill records (i.e., picking up each new PrEP supply), and self-report (e.g., self-rating, captured on the PrEP delivery tool).

\section{Nested observational cohort for individual-level outcomes}

Within the programmatic setup, we will establish an open cohort of HIV-uninfected women to measure individuallevel outcomes on incident HIV and STIs, HIV prevention behavior, PrEP use, and adherence. The cohort will enroll up to 900 women, including women who do and do not choose to initiate PrEP. Eligible women must be HIV negative, sexually active, age 15 years and older, and have at least one behavioral factor defined by the Kenya
PrEP guidelines to indicate a substantial ongoing risk of acquiring HIV (as described in the Subsection on Screening for HIV risk and PrEP). Informed consent will be obtained for all research procedures not related to routine care. Cohort participants will complete visits at one month and then quarterly after enrollment for up to 24 months with HIV testing and a brief questionnaire on sexual behaviors, FP and prevention methods use, HIV and STI risk, partner relationship, stigma, mental health, and for women initiated on PrEP, dried blood spots will be collected for objective assessment of adherence. All cohort activities will be conducted by project-dedicated staff. In addition to promotion of standard screening and treatment for STIs across all clinics, we will perform baseline etiological testing for curable STIs (Chlamydia trachomatis and Neisseria gonorrhea) to document the burden for women enrolled in the cohort. Urine from cohort follow-up visits will be archived for future STI testing.

\section{Health economics studies}

We will conduct activity-based costing, staff interviews, and time and motion studies to estimate the cost and model the budget impact of integrating PrEP provision in FP clinics on annual basis, over a 5-year horizon. Using data on the incremental cost estimated during the implementation phase, we will assess the budget impact on the Kenya $\mathrm{MOH}$ and Kisumu County budgets for integrating the interventions incorporating the eligible population size, and current $\mathrm{MOH}$ and Kisumu budget expenditures, and evaluate for scenarios with current and future HIV prevention methods mix. We will consider direct program costs to ensure that measurements of $\mathrm{MOH}$ costs reflect the opportunity cost of the resources used in delivering services. Program costs will also be collected from the project budget, public health clinic budgets, government reports, and the health economics literature, as we have done in previous work [27-30]. The primary analysis will be from the programmatic perspective, with a secondary analysis from the societal perspective to account for client opportunity, affordability, and financial costs. Health outcomes will include incident HIV cases and disability-adjusted life years averted.

\section{Data management and confidentiality}

All abstracted health and patient data are kept secure and confidential through adherence to institutional policies and procedures for securely storing, maintaining, and updating health record information. All health record and patient data are securely stored on password protected and encrypted servers and these data will not be released externally except under specific data sharing agreements. All study results will be presented in 
aggregate, and no individual patient or provider will be identifiable.

\section{Analysis \\ Quantitative data:}

The primary analysis will use individual-level data from the SWCRT (counseling on HIV risk and PrEP, PrEP initiation, provision of contraception, and type of contraception provided), individual-level data from the blood sample cohort (PrEP adherence), individual-level data from the longitudinal research cohort, and clinic-level data from the SWCRT (HIV diagnosis). Descriptive statistics of the outcomes will be summarized by study step (baseline, step one, step two or step three) and clinic within each step, as well as overall.

\section{Outcomes and Inference}

The co-primary outcome to evaluate the intervention effect will be: 1) proportion of women screened for on HIV risk and PrEP; and 2) PrEP uptake. Analyses of the remaining outcomes (knowledge of partner HIV status, PrEP adherence, HIV diagnosis, provision of contraception, type of contraception provided, and STI screening and treatment) will be descriptive and will only involve individuals enrolled (for individual-level outcomes) and data collected (for clinic-level outcomes) after the intervention was initiated. The relative risk (RR) for the effect of the intervention on each outcome will be estimated from the individual-level data (based on baseline and steps one to three) using a generalized estimating equations (GEE) approach [31, 32]. Specifically, we will fit a modified Poisson GEE, i.e., a generalized linear model with log link and robust standard errors [33] to account for clinic clustering in outcomes and to appropriately model a binary outcome, allowing estimation of the relative risk. The predictor of interest will be the intervention status of the site at the time the participant was enrolled (control or intervention). The model will adjust for the partial confounding by time inherent in this design by including the step during which the participant enrolled as a categorical variable in the model. Using the implementation and maintenance phases, we will conduct secondary analyses using similar methods to estimate changes in both clinical and implementation outcomes during the maintenance phase compared to the intensive phase to quantitatively ascertain level of institutionalization. Importantly, we will use data from the maintenance phase to descriptively explain implementation outcomes. In sensitivity analyses, we will also repeat the above analysis but allow for the intervention effect to vary with time; in other words, we will include an interaction between treatment assignment and step, where step is modeled as a categorical variable [34]. This will allow us to evaluate possible variations in treatment effect over time given that the intervention may change over time due to feedback throughout the study period.

\section{Qualitative data}

Qualitative data (i.e., technical assistance reports, individual interviews, and observation reports) will be used to interpret and explain contextual influences on individual RE-AIM dimensions or patterns of results across implementation phases and/or clinics. We will use rapid qualitative analysis approaches to obtain real-time information [35-37] that can directly inform study implementation. In addition, we will use a combination of conventional and directed content analysis approaches [38] to identify determinants of high and low performing facilities during early implementation and maintenance phases. Coding and analysis will be performed in Atlas.ti. Qualitative data sources (transcripts and reports) will be iteratively coded using a structured codebook developed deductively from the constructs within CFIR and inductively from open-coding. Coding will be conducted by multiple team members, and each data source reviewed by at least two members of the research team to ensure consistency of text segmentation and code application. Queries will be used to abstract related concepts and generate preliminary themes. Preliminary themes will be grouped together into larger themes through group deliberative processes. We will triangulate qualitative and quantitative data together to answer relevant questions about delivery process and outcomes simultaneously. Specially, we will characterize common themes and profiles of low and high performing facilities as determined by the proportion of women screened, initiated, and retention on PrEP.

\section{Protocol amendments}

This protocol (version 1.0, October 27, 2020) was registered with ClinicalTrials.gov on December 14, 2020 (NCT04666792). Amendments to the study protocol will require approval from the Ethics Review Committee of the Kenyatta National Referral Hospital- University of Nairobi and the Human Subjects Division of the University of Washington. Any amendments will be communicated via trial registration updates and reported in any published manuscripts associated with the study as necessary.

\section{Dissemination plan}

The study team for this award is committed to public dissemination of results of the project to local stakeholders in Kenya, the global scientific community, and global policymakers. Dissemination of study results will follow principles of good participatory practice. Results will 
be published in conference abstracts and peer-reviewed journals. Study results will be disseminated through presentations to global and local stakeholders and policymakers in Kenya, including working in close collaboration with the Kenyan Ministry of Health to help foster immediate translational impact. Authorship eligibility will be determined according to the International Committee of Medical Journal Editors recommendations.

\section{Project status}

The project activated in March 2021 and implementation is currently ongoing and expected to end in 2024.

\section{Discussion}

As PrEP implementation gradually expands in Africa, initial delivery points have centered in HIV care centers focused on serodiscordant couples, "drop-in" centers, and youth-friendly "safe spaces," for key populations. For women, access has been limited mostly through a few demonstration projects in FP and antenatal clinics.

The FP Plus project offers a timely opportunity to move from small-scale PrEP demonstration projects to fullscale service delivery for women in Africa. Since the 2015 WHO global recommendation for PrEP was released, understanding how to rapidly and efficiently translate this effective prevention intervention into real-world settings has been the priority, but most work in Africa remains focused on controlled demonstration projects, mostly in research clinics. We have pushed beyond this boundary with the PrIYA project, a demonstration pilot project that has shown that it may be feasible to integrate PrEP in FP systems. In this study we move to the next phase and evaluate whether fully integrating PrEP provision in FP clinics as a one-stop location for women-centered health care services can be done successfully at scale with implementation science evaluations. Our study leverages existing infrastructure and expertise of FP providers in counseling for sexual history and program delivery on a variety of contraception methods, to embed clinical assessment and PrEP provision in combination HIV prevention package. Using current health system, staffing and supply chain and has tremendous potential to address barriers that women face for PrEP access, including lack of time, cost, and stigma of visiting a facility solely for HIV prevention. With more efficient PrEP integration, there is opportunity to improve FP care and optimize women-centered care with a one-stop approach to clinic. The implementation methods and lessons learned through our novel FP-based PrEP care pathway will provide information on sustainable integrated service delivery that can be translated to other African settings. Importantly, the application of rigorous implementation science approaches to understand implementation will be valuable for advancing other emerging novel HIV prevention modalities into real-world delivery.

In Kenya's devolved government structure, the $\mathrm{MOH}$ sets and guides national policy, but implementation of policy and public health services is under the county governments. Our proposed work aligns with the Kenya $\mathrm{MOH}$ and Kisumu County priority goals, which makes the probability of impact high. In 2016, Kenya $\mathrm{MOH}$ developed a national PrEP implementation framework [21], and national PrEP roll-out was officially launched in May 2017, making it the first African national PrEP program. Because of the Kenyan governments' strong support for PrEP as an HIV prevention intervention, Kenya is an incubator for research on innovative PrEP delivery models. Successful implementation of PrEP interventions in Kenya has the potential to influence the delivery of PrEP in other African settings. This work will inform implementation of similar interventions elsewhere and facilitate interpretation of intervention outcomes.

\section{Abbreviations \\ ART: Antiretroviral therapy; CFIR: Consolidated framework for implementation science research; DMC: Data monitoring committee; FP: Family planning; HIV: Human immunodeficiency virus; KEMSA: Kenya medical supply authority; $\mathrm{MOH}$ : Ministry of Health; NASCOP: National AIDS \& STI control programme; PrEP: Pre-exposure prophylaxis; PrIYA: PrEP implementation in young women and adolescent girls; RE-AIM: Reach, effectiveness, adoption, implementation, maintenance; SRH: Sexual and reproductive health; STI: Sexually transmitted infection; SWCRT: Stepped-wedge cluster-randomized trial; TA: Technical advisors; TWG: Technical working group; WHO: World health organization.}

\section{Acknowledgement}

We thank the women who are participating in this project and the staff at implementing facilities and the project staff for their motivation and dedication.

\section{Authors' contributions}

$\mathrm{KKM}, \mathrm{JK}, \mathrm{AM}, \mathrm{KB}, \mathrm{BW}, \mathrm{JMB}$ contributed to the conception and design of the project; KKM, with JK, prepared the first draft of this manuscript. DM, CS, $\mathrm{KB}, \mathrm{DO}, \mathrm{MM}, \mathrm{AM}, \mathrm{EF}, \mathrm{CG}, \mathrm{RB}, J \mathrm{MB}, \mathrm{BW}$ contributed to the writing and critical revision of the paper. All authors read and approved the final manuscript. For questions about the FP Plus project contact the International Clinical Research Center at the University of Washington (icrc@uw.edu).

\section{Funding}

The FP Plus Project is supported by the National Institute of Mental Health of the US National Institutes of Health (grant R01 MH123267 and R00

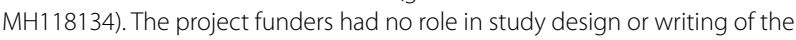
report.

\section{Availability of data and materials}

The datasets generated from this protocol will be available after the primary analysis by contacting the International Clinical Research Center at the University of Washington (icrc@uw.edu).

\section{Declarations}

Ethics approval, consent to participate and monitoring

The protocol described herein was approved as a minimal risk study by the Ethical Research Committee of the Kenyatta National Referral Hospital and the Human Subjects Division of the University of Washington. As is the case for all routine services, oral consenting for routine services (i.e., all procedures key for 
provision of PrEP as part of standard of care, HIV testing, and PrEP provision) will be done by the existing FP healthcare providers. Persons who participate in all planned research related procedures (procedures not directly related to standard of care provision of PrEP services e.g., qualitative interviews, questionnaires, blood draw for drug levels, participation in observational cohort, archiving of specimens for STI testing), written informed consent will be obtained before any procedures are conducted. Consenting for research procedures will be conducted by the research team. The research team meets weekly for executive as well as safety and quality management purposes. The project is subject to oversight by an independent external data monitoring committee (DMC) established to oversee project implementation, data quality, monitor implementation and clinical outcomes, and patient safety. The committee consists of expert clinicians, statisticians, policymakers and stakeholders from the HIV and SRH space in Kenya settings. The committee meets 6-monthly to advise on study progress and whether the work aligns with Kenya $\mathrm{MOH}$ policies, and to offer guidance about results from international studies of PrEP that may spur policy change in Kenya. The DMC will review data on HIV testing and counseling, HIV infection, FP service use, PrEP uptake, continue and adherence, adverse outcomes, implementation outcomes, and will provide recommendations to the project implementation team as part of six-monthly reviews. Review follow will follow an unblinded fashion, consistent with the open label, unblinded nature of the implementation project. No interim analyses are planned for this open-label implementation project.

\section{Consent for publication}

Not applicable

\section{Competing interests}

All authors declare no competing interests

\section{Author details}

'Departments of Global Health and Epidemiology, University of Washington, 325 Ninth Avenue, \#HMC 359927, Seattle, WA 98104, USA. ${ }^{2}$ Research \& Programs, Kenyatta National Hospital, Nairobi, Kenya. ${ }^{3}$ Department of Global Health, University of Washington, Seattle, USA. ${ }^{4}$ Vaccine and Infectious Diseases Division, Fred Hutchinson Cancer Research Center, Seattle, USA. ${ }^{5}$ Kisumu County Department of Health, Kisumu, Kenya. ${ }^{6}$ National AIDS and STI Control Program Ministry of Health, Nairobi, Kenya. ${ }^{7}$ Departments of Global Health and Medicine, University of Washington, Seattle, USA. ${ }^{8}$ Departments of Global Health, Medicine, and Epidemiology, University of Washington, Seattle, USA. ${ }^{9}$ Present affiliation: Gilead Sciences, Foster City, USA. ${ }^{10}$ Kenyatta National Referral Hospital, Nairobi, Kenya.

\section{Received: 28 September 2021 Accepted: 15 October 2021} Published online: 11 December 2021

\section{References}

1. UNAIDS. UNAIDS data 2018; 2018 [updated 2018; cited 2019 September 03]; Available from: https://www.unaids.org/en/resources/documents/ 2018/unaids-data-2018.

2. Baeten JM, Donnell D, Ndase P, Mugo NR, Campbell JD, Wangisi J, et al. Antiretroviral prophylaxis for HIV prevention in heterosexual men and women. N Engl J Med. 2012;367(5):399-410 PMCID: 3920826.

3. Choopanya K, Martin M, Suntharasamai P, Sangkum U, Mock PA, Leethochawalit M, et al. Antiretroviral prophylaxis for HIV infection in injecting drug users in Bangkok, Thailand (the Bangkok Tenofovir Study): a randomised, double-blind, placebo-controlled phase 3 trial. Lancet. 2013;381(9883):2083-90.

4. Grant RM, Lama JR, Anderson PL, McMahan V, Liu AY, Vargas L, et al. Preexposure chemoprophylaxis for HIV prevention in men who have sex with men. N Engl J Med. 2010;363(27):2587-99.

5. Kenya Ministry of Health. Demographic andHealth Survey; 2014 [updated 2014; cited 2019 August 30]; Available from: https://dhsprogram.com/ pubs/pdf/fr308/fr308.pdf.

6. O. Evidence for Contraceptive and H. I. V. O. T. Consortium. HIV incidence among women using intramuscular depot medroxyprogesterone acetate, a copper intrauterine device, or a levonorgestrel implant for contraception: a randomised, multicentre, open-label trial. Lancet. 2019;394(10195):303-13 PMCID: PMC6675739.
7. United Nations. The Millennium Development Goals Report; 2010 [updated 2010; cited 2019 September 03]; Available from: http://mdgs. un.org/unsd/mdg/Resources/Static/Products/Progress2010/MDG_ Report_2010_En.pdf.

8. Mugwanya KK, Pintye J, Kinuthia J, Abuna F, Lagat H, Begnel ER, et al. Integrating preexposure prophylaxis delivery in routine family planning clinics: a feasibility programmatic evaluation in Kenya. PLoS Med. 2019;16(9):e1002885.

9. Kenya Ministry of Health. Kenya HIV prevention revolution road map: Count down to 2030; 2014 [updated 2014; cited 2017 December 12]; Available from: http://www.nacc.or.ke/images/documents/Final.pdf.

10. Kenya Ministry of Health. Guidelines on the use of antiretroviral drugs for treating and preventing HIV infection in Kenya; 2016 [updated 2016; cited 2017 December 12]; Available from: http://emtct-iatt.org/wp-conte nt/uploads/2016/09/Guidelines-on-Use-of-Antiretroviral-Drugs-for-Treat ing-and-Preventing-HI....pdf.

11. Baeten JM, Donnell D, Mugo NR, Ndase P, Thomas KK, Campbell JD, et al. Single-agent tenofovir versus combination emtricitabine plus tenofovir for pre-exposure prophylaxis for HIV-1 acquisition: an update of data from a randomised, double-blind, phase 3 trial. Lancet Infect Dis. 2014;14(11):1055-64 PMCID: PMC4252589.

12. Donnell D, Baeten JM, Bumpus NN, Brantley J, Bangsberg DR, Haberer JE, et al. HIV protective efficacy and correlates of tenofovir blood concentrations in a clinical trial of PrEP for HIV prevention. J Acquir Immune Defic Syndr. 2014;66(3):340-8 PMCID: PMC4059553.

13. Donnell D, Baeten JM, Kiarie J, Thomas KK, Stevens W, Cohen CR, et al. Heterosexual HIV-1 transmission after initiation of antiretroviral therapy: a prospective cohort analysis. Lancet. 2010;375(9731):2092-8 PMCID: PMC2922041.

14. Heffron R, Mugo N, Were E, Kiarie J, Bukusi EA, Mujugira A, et al. Preexposure prophylaxis is efficacious for HIV-1 prevention among women using depot medroxyprogesterone acetate for contraception. AIDS. 2014;28(18):2771-6 PMCID: PMC4266161.

15. Ngure K, Heffron R, Curran K, Vusha S, Ngutu M, Mugo N, et al. Experiences of Kenyan HIV serodiscordant couples soon after pre-exposure prophylaxis (PrEP) initiation. Aids Patient Care Stds. 2016;30(2):78-83.

16. Baeten JM, Donnell D, Ndase P, Mugo NR, Campbell JD, Wangisi J, et al. Antiretroviral prophylaxis for HIV prevention in heterosexual men and women. N Engl J Med. 2012;367(5):399-410.

17. Mugwanya K, Baeten J, Celum C, Donnell D, Nickolas T, Mugo N, et al. Low risk of proximal tubular dysfunction associated with emtricitabine-tenofovir disoproxil fumarate preexposure prophylaxis in men and women. J Infect Dis. 2016.

18. Mugwanya KK, Wyatt C, Celum C, Donnell D, Kiarie J, Ronald A, et al. Reversibility of glomerular renal function decline in HIV-uninfected men and women discontinuing emtricitabine-tenofovir disoproxil fumarate pre-exposure prophylaxis. J Acquir Immune Defic Syndr. 2016;71(4):37480 PMCID: PMC4770857.

19. Mugwanya KK, Wyatt C, Celum C, et al. Changes in glomerular kidney function among hiv-1-uninfected men and women receiving emtricitabine-tenofovir disoproxil fumarate preexposure prophylaxis: A randomized clinical trial. JAMA Intern Med. 2015;175(2):246-54

20. Mugwanya KK, Hendrix CW, Mugo NR, Marzinke M, Katabira ET, Ngure $K$, et al. Pre-exposure prophylaxis use by breastfeeding HIV-uninfected women: a prospective short-term study of antiretroviral excretion in breast milk and infant absorption. PLOS Med. 2016;13(9):e1002132.

21. Kenya Ministry of Health. Framework for Implementation of Pre-Exposure Prophylaxis of HIV in Kenya; 2017 [updated 2017; cited]; Available from: Available at: http://www.prepwatch.org/wp-content/uploads/2017/05/ Kenya_PrEP_Implementation_Framework.pdf. Accessed August 03, 2017.

22. Shea CM, Jacobs SR, Esserman DA, Bruce K, Weiner BJ. Organizational readiness for implementing change: a psychometric assessment of a new measure. Implementation Sci. 2014;9(1):7.

23. Damschroder LJ, Aron DC, Keith RE, Kirsh SR, Alexander JA, Lowery JC. Fostering implementation of health services research findings into practice: a consolidated framework for advancing implementation science. Implementation Sci. 2009;4(1):50.

24. Grol R, Grimshaw J. From best evidence to best practice: effective implementation of change in patients' care. Lancet. 2003;362(9391):1225-30.

25. Rowe AK, de Savigny D, Lanata CF, Victora CG. How can we achieve and maintain high-quality performance of health workers in low-resource settings? Lancet. 2005;366(9490):1026-35. 
26. Fernandez ME, Walker TJ, Weiner BJ, Calo WA, Liang S, Risendal B, et al. Developing measures to assess constructs from the Inner Setting domain of the Consolidated Framework for Implementation Research. Implement Sci. 2018;13(1):52 PMCID: PMC5870186.

27. Golovaty I, Sharma M, Van Heerden A, van Rooyen H, Baeten JM, Celum $C$, et al. Cost of integrating noncommunicable disease screening into home-based HIV testing and counseling in South Africa. J Acquir Immune Defic Syndr. 2018;78(5):522-6 PMCID: PMC6037552.

28. Irungu EM, Sharma M, Maronga C, Mugo N, Ngure K, Celum C, et al. The incremental cost of delivering PrEP as a bridge to ART for HIV serodiscordant couples in public HIV care clinics in Kenya. AIDS Res Treat. 2019;2019:4170615 PMCID: PMC6521338.

29. Phillips AN, Cambiano V, Nakagawa F, Bansi-Matharu L, Wilson D, Jani I, et al. Cost-per-diagnosis as a metric for monitoring cost-effectiveness of HIV testing programmes in low-income settings in southern Africa: health economic and modelling analysis. J Int AIDS Soc. 2019;22(7):e25325 PMCID: PMC6615491.

30. Roberts DA, Barnabas RV, Abuna F, Lagat H, Kinuthia J, Pintye J, et al. The role of costing in the introduction and scale-up of HIV pre-exposure prophylaxis: evidence from integrating PrEP into routine maternal and child health and family planning clinics in western Kenya. J Int AIDS Soc. 2019;22(Suppl 4):e25296 PMCID: PMC6643078.

31. Hughes JP, Granston TS, Heagerty PJ. Current issues in the design and analysis of stepped wedge trials. Contemp Clin Trials. 2015;45(Pt A):55-60 PMCID: PMC4639463.

32. Hussey MA, Hughes JP. Design and analysis of stepped wedge cluster randomized trials. Contemp Clin Trials. 2007;28(2):182-91.

33. Yelland LN, Salter AB, Ryan P. Performance of the modified Poisson regression approach for estimating relative risks from clustered prospective data. Am J Epidemiol. 2011;174(8):984-92.

34. Hemming K, Eldridge S, Forbes G, Weijer C, Taljaard M. How to design efficient cluster randomised trials. BMJ. 2017;358:j3064.

35. Simoni JM, Beima-Sofie K, Amico KR, Hosek SG, Johnson MO, Mensch $B S$. Debrief reports to expedite the impact of qualitative research: do they accurately capture data from in-depth interviews? AIDS Behav. 2019;23(8):2185-9 PMCID: PMC6642848.

36. Skillman M, Cross-Barnet C, Friedman Singer R, Rotondo C, Ruiz S, Moiduddin A. A framework for rigorous qualitative research as a component of mixed method rapid-cycle evaluation. Qual Health Res. 2019:29(2):279-89.

37. Vindrola-Padros C, Johnson GA. Rapid techniques in qualitative research: a critical review of the literature. Qual Health Res. 2020;30(10):1596-604.

38. Hsieh HF, Shannon SE. Three approaches to qualitative content analysis. Qual Health Res. 2005;15(9):1277-88.

\section{Publisher's Note}

Springer Nature remains neutral with regard to jurisdictional claims in published maps and institutional affiliations.

Ready to submit your research? Choose BMC and benefit from:

- fast, convenient online submission

- thorough peer review by experienced researchers in your field

- rapid publication on acceptance

- support for research data, including large and complex data types

- gold Open Access which fosters wider collaboration and increased citations

- maximum visibility for your research: over $100 \mathrm{M}$ website views per year

At BMC, research is always in progress.

Learn more biomedcentral.com/submissions 